\title{
Application of matlab ordinary differential equation function solver (ode45) in modelling and simulation of batch reaction kinetics
}

\author{
${ }^{1}$ Anyigor, C.M, and ${ }^{1}$ Afiukwa, J.N \\ ${ }^{1}$ Department of Industrial Chemistry, Ebonyi State University, P.M.B. 053 Abakaliki, Ebonyi \\ State, Nigeria. Email: chumaco_15@yahoo.com
}

\begin{abstract}
This study presents the simulation of modelled chemical reaction in a batch reactor using the Matlab ordinary differential equation function solver (ODE45). Modelling and optimisation of actual batch reaction rate constants under isothermal condition was implemented on a set of experimental data. The data was simulated using hypothetical isothermal rate constants, $\mathrm{K}_{1}(0.5)$ and $\mathrm{K}_{2}(0.1)$ with the initial concentration profile of the reaction: $\mathrm{A}=\mathrm{B}=1$ and $\mathrm{C}=\mathrm{D}=$ $\mathrm{E}=0$ respectively between reaction time of $0-40$ minutes. The Process model developed was employed to optimise the rate constant $(K)$ at which maximum yield of the desired product was achieved. Results showed maximum product yield, ' $D$ ' at $K_{1=} 0.5$ with a reaction by-product, 'E' at $\mathrm{K}_{2}=0.1$ within 40 minutes reaction time. This study is intended to benefit industrial operations which aim at achieving optimum yield of a reaction process in a record time.
\end{abstract}

Keywords: Reaction Kinetics, Simulation, Modelling, Matlab and Process control.

\section{INTRODUCTION}

The focus of an industrialist is how to achieve maximum yield of the desired product in a reasonable time frame. Chemical Kinetics is concerned with the velocity or time rate of reaction and the mechanisms of the reaction. The rate constant determines how quantitative the yield of the reaction is. Therefore efforts aimed at optimising the rate constant would enhance yield maximally. Computer aided mathematical application is a growing trend used in modelling and simulation of reaction kinetics to achieve such a desired optimum yield (Ullah and Wolkenhauer 2011). In many chemical processes of transformation of reactants into products, only the disappearance of the reactants and appearance of the final products may be observed (Sharma and Pathania 2006). But for batch reactions occurring in steps, intermediate products may be formed which may not be detected because they are promptly used up in the subsequent step to yield the main product. The rate of reaction generally decreases with time but the coefficient of determination (rate constant) remains unchanged throughout the reaction. The rate depends on the active concentration of the reactants and is greatly influenced by temperature among other factors such as the nature and concentration of the reacting species (Higham 2007).
In this study, the use of process model of a hypothetical batch reaction of the type: $A+B \stackrel{K_{1}}{\rightarrow} C+$ $D \ldots .$. (1) and $C+A \stackrel{K_{2}}{\rightarrow} E \ldots$ (2), was implemented through the application of Matlab ordinary differential equation (ODE45) function solver in order to optimise the rate constants $\left(k_{\max }\right)$ under isothermal condition. Certain relevant assumptions were made and hypothetical K-values grouped as $K_{1}, K_{2}=$ $0.5,0.1 ; 0.2,0.4$ and $1.0,2.0$ respectively were investigated on the hypothetical reaction equations to find the optimum $\mathrm{K}\left(\mathrm{k}_{\max }\right)$.

\section{METHODOLOGY:}

The initial conditions of the concentration species used were; $\quad C_{A}=1, C_{B}=1, C_{C}=C_{D}=C_{E}=0$ respectively and the following assumptions associated with batch reactions occurring in a reactor were made: (i) that the batch reactor is a homogeneous mixture, (ii) operate isothermally under closed system, (iii) has constant volume and (iv) occur at the rates, $r_{1}$ and $r_{2}$ for the hypothetical reactions 1 and 2 respectively. The material balance of the batch reactor was obtained from the general equation:

Rate of Change $=$ Flow in $\left(F_{\text {in }}\right)-$ Flow out $\left(F_{\text {out }}\right)+$ Generation - consumption

Where for a batch reactor, $F_{\text {in }}-F_{\text {out }}=0$; then Rate of Change $=$ Generation - consumption 


$$
\begin{aligned}
& r_{1}=K_{1} C_{A} C_{B} \\
& r_{2}=K_{2} C_{A} C_{C}
\end{aligned}
$$

The numerical differential equations of the reaction rates were defined based on the material balance and integrated using the ODE45 solver function of Matlab (Howard 2007). The developed differential models of the reaction rates for each of the reaction parameters (A, B, C, D and E) were shown:

$$
\begin{array}{r}
\frac{d C_{A}}{d t}=-r_{1}-r_{2}=-K_{1} C_{A} C_{B}-K_{2} C_{A} C_{C} \\
\frac{d C_{B}}{d t}=-r_{1}=-K_{1} C_{A} C_{B}
\end{array}
$$

$$
\begin{array}{r}
\frac{d C_{C}}{d t}=r_{1}-r_{2}=K_{1} C_{A} C_{B}-K_{2} C_{A} C_{C} \\
\frac{d C_{D}}{d t}=r_{1}=K_{1} C_{A} C_{B}
\end{array}
$$

$$
\frac{d C_{E}}{d t}=r_{2}=K_{2} C_{A} C_{C}
$$

Where $C_{A}, C_{B}, C_{c}, C_{D} \& C_{E}$ were the concentration species, $C_{A} \& C_{B}$ were the reactants, $C_{C}$ was the intermediate product whereas $C_{D} \& C_{E}$ were the final products occurring in the batch reactor. $K_{1}$ and $K_{2}$ were the isothermal kinetic reaction rate coefficient for reaction 1 and 2 respectively.

Two Matlab files were created. The main file ( $m$-file) of Matlab code of the initial condition of concentration species put in vector form was used to define the initial conditions and to call up the second Matlab file of codes (function file), '[t,y] = ode45(@reactor1,[0 40],y)'. The function file was employed by integrating the ODEs of the reaction model (Appendices $1-4$ ). Simulation was implemented and the results of concentration against time were plotted. The simulation using the ODE45 function solver of Matlab solved the integration of the established reaction models of the batch reactor system from initial time to the final time conditions of $0-40$ minutes. The integral time limits adopted in this work was realised by trial and error until a constant concentration profile was obtained

\section{RESULTS AND DISCUSSION}

Application of Matlab ODE45 solver function on hypothetical bimolecular reaction kinetics to optimise the rate constants was investigated under isothermal condition. In the hypothetical bimolecular reaction: $A+B \stackrel{K_{1}}{\rightarrow} C+D ; C$ served as an intermediate product which instantly reacted with $A$ to yield $E$ as a by- product of the net reaction i.e. $C+A \stackrel{K_{2}}{\rightarrow} E$. Thus reactant $A$ was totally consumed as indicated with its concentration dropping to zero from unity (Fig.3).

Based on the reaction models implementation (Appendices 1-4) and the integrated numerical differential equations of the reaction rates (Equations $3-7$ ) within limits of $0-40$ minutes, the results of the plot of concentration $\left(\mathrm{mol} / \mathrm{dm}^{3}\right)$ of the species involved in the reaction against time (mins) were shown in Figs 1, 2, and 3 . The batch reactor was allowed to progress for 40 minutes to attain a constant concentration profile of species indicated as legends in the figures. Maximum value of the main product, ' $D$ ', $\left(0.83 \mathrm{~mol} / \mathrm{dm}^{3}\right)$ was realised between $16-40$ minutes at an assumed $K_{1}$ and $K_{2}$ values of 0.5 and 0.1 respectively (Fig.3). The concentration of ' $D$ ' rose from 0.0 and levelled up at $0.83 \mathrm{~mol} / \mathrm{dm}^{3}$ from 16-40 minutes. After this time limit, it is unlikely to have more yields since one of the reactants seem to have been totally consumed. This value was much higher than was obtained at $\mathrm{K}_{1}$ and $\mathrm{K}_{2}$ tried at 1.0 and 2.0 (Fig 1) and 0.2 and 0.4 (Fig. 2) respectively. The result of Fig. 1 is unthinkable, meaning that positive integer values of the rate constants may not yield reasonable product. The relationship between Figs. 2 and 3 indicated that there should be an optimum K-value at which maximum yield of the desired product can be achieved. This optimum rate constant was attained at $K_{1}=5 \times 10^{-1}$ referring to equation 1 . The $K_{2}=1 \times$ $10^{-1}$ becomes useful if the side reaction giving the by- product ' $E$ ' was desired. Negative values of $K$ at 1 and -2 were also evaluated but the result was negative implying that rate constant cannot be negative.

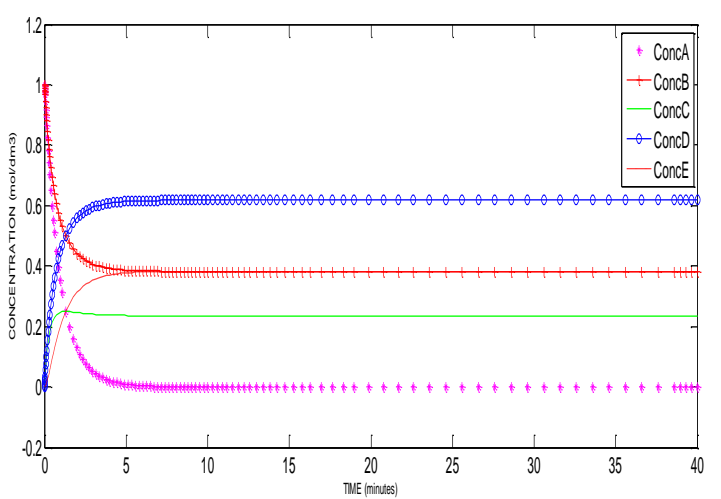

Fig. 1: Concentration Profile of the Batch Reactor after reaction time of $\mathbf{4 0}$ minutes with rate constant values of 1.0 and 2.0. 


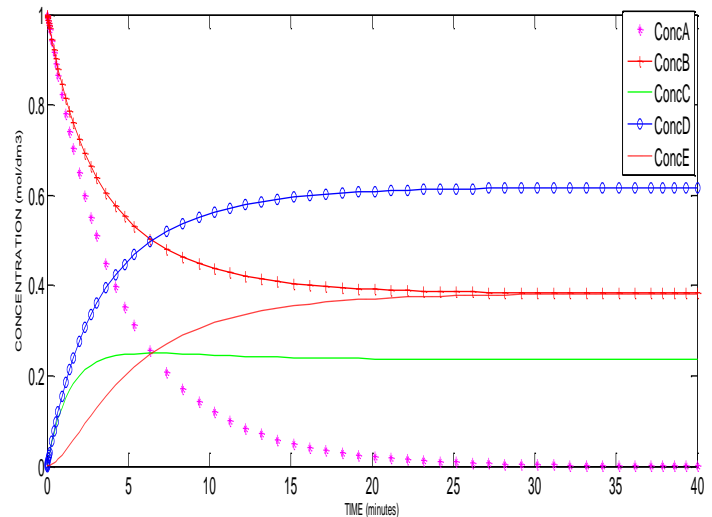

Fig. 2: Concentration Profile of the Batch Reactor after reaction time of $\mathbf{4 0}$ minutes with rate constant values of 0.2 and 0.4 .

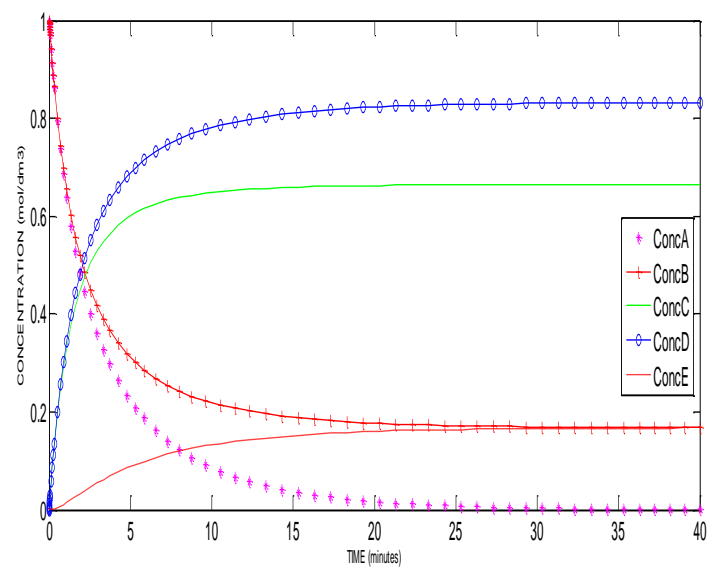

Fig. 3: Concentration Profile of the Batch Reactor after reaction time of $\mathbf{4 0}$ minutes with a rate constant values of 0.5 and 0.1 .

\section{CONCLUSION}

Simulation of kinetic process models using Matlab functions solver, ODE45 programme proved to be a reliable finger print for obtaining dependable results of Industrial Chemical Processes. The results obtained in this study showed that maximum yield of the product ' $D$ ' $\left(0.83 \mathrm{~mol} / \mathrm{dm}^{3}\right)$ was obtained when the coefficient of determinant, $\mathrm{K}_{1}$ of the reaction was $5 \times$ $10^{-1}$. This maximum result was achieved within an optimised time frame of $16-40$ minutes under constant temperature. The order of the reaction can be estimated from the reaction rate constant equation which gave the $\mathrm{K}_{\max }$.

\section{RECOMMENDATION}

The Matlab ODE45 function solver can be tried on complex reactions such as reversible $A \rightleftharpoons B$; consecutive $(A \stackrel{K 1}{\rightarrow} B \stackrel{k 2}{\rightarrow} C)$ and parallel $(A \stackrel{k 1}{\rightarrow} B ; A \stackrel{k 2}{\rightarrow} C)$. It is also recommended for chemical, biological and biochemical reaction systems for batch reactor system analysis and product yield maximization.

\section{Acknowledgement}

The authors acknowledge with thanks Dr. Mark Willis, Senior Lecturer, Applied Process Control Newcastle University UK, for his invaluable instructional mentoring that inspired this line of thought.

\section{REFERENCE}

Denisov, T. E., O. M. Sarkisov, et al. (2003). Chemical Kinetics: Fundamentals and New Developments. Amsterdam, Netherland, ELSERVIER SCIENCE B. V.

Higham, D. J. (2007). Modelling and Simulation Chemical Reactions. Available at: https://www.google.com.ng/webhpsource=search app\#hl=en\&gs $r n=9 \& g s \quad r i=p s y$ ab\&tok=0dWmG3ID0NXRDUIJIKW6Ag\&cp $=59 \& \mathrm{~g}$ $\mathrm{s}$ id $=72 \& x h r=t \& q=$ chemical+kinetics+reaction+rat e+modelling+with+Matlab+ODE45\&es nrs=true\& $\mathrm{pf}=\mathrm{p} \&$ sclient $=\mathrm{psyab \& oq}=$ chemical+kinetics + reacti on+rate+model

Howard, P. (2007). Solving ODE in MATLAB. Avaliable at: www.cs.rice.edu/../matode.pdf (Accessed August 2012): Pages 1 - 22.

Sharma, L. R. and M. S. Pathania (2006). PRINCIPLES OF PHYSICAL CHEMISTRY. Jalandhar, India, VISHAL PUBLISHING CO.

Ullah, M. and O. Wolkenhauer (2011). Stochastic Approaches for Systems Biology. Available at: http://www.springer.com/978-1-4614-0477-4, Springer. 290: p. 73. 